\title{
Etre pris dans le mouvement. Savoir et engagement sur le terrain. Partie 1
}

\section{Elise Massicard}

\section{(2) OpenEdition \\ 1 Journals}

Édition électronique

URL : http://journals.openedition.org/conflits/838

DOI : $10.4000 /$ conflits. 838

ISSN : $1777-5345$

\section{Éditeur :}

CCLS - Centre d'études sur les conflits lilberté et sécurité, L'Harmattan

\section{Édition imprimée}

Date de publication : 1 septembre 2002

ISBN : 2-7475-3432-4

ISSN : 1157-996X

\section{Référence électronique}

Elise Massicard, «Etre pris dans le mouvement. Savoir et engagement sur le terrain. Partie 1 », Cultures \& Conflits [En ligne], 47 | automne 2002, mis en ligne le 29 avril 2003, consulté le 30 mars 2021. URL : http://journals.openedition.org/conflits/838 ; DOI : https://doi.org/10.4000/conflits.838

Ce document a été généré automatiquement le 30 mars 2021.

Creative Commons License 


\title{
Etre pris dans le mouvement. Savoir et engagement sur le terrain. Partie 1
}

\author{
Elise Massicard
}

Comment un terrain devient-il «difficile»? Les réponses à cette question relèvent d'appréciations inévitablement subjectives. Nous considérerons dans cet article la « difficulté » au sens large, c'est-à-dire non seulement comme danger physique, mais aussi comme la gestion problématique des situations concrètes d'enquête et d'interaction. Plus précisément, nous interviendrons sur la dimension «politique » de l'inconfort propre à la situation de terrain, dans laquelle le chercheur "s'expose " nécessairement. En effet, si la démarche scientifique requiert une distance analytique et une neutralité normative, les enquêtés réclament parfois un engagement en leur faveur. Dans la situation d'enquête qui a été la nôtre pendant plusieurs années ${ }^{1}$, non seulement les enquêtés - des entrepreneurs d'un mouvement identitaire -, mais aussi les autorités ont effectué des projections sur le chercheur, demandant, orientant, limitant, voire refusant son "intrusion ». Ces demandes sociales émanant d'horizons différents ont conditionné le déroulement même de l'enquête, menant à des positionnements qui peuvent être considérés, d'un point de vue déontologique, comme autant de « compromissions ».

Dans une réflexion sur l'exposition du chercheur sur son terrain, la question de sa multipositionnalité nous paraît centrale : pour mener à bien son travail, il doit (feindre de) répondre à différentes demandes, jongler avec diverses projections, gérer des "dédoublements", mais aussi reconnaître l'existence de limites parfois infranchissables. Souvent surprenantes, les difficultés rencontrées dans la gestion de ces tensions sont susceptibles d'entrâner l'évolution dialectique des stratégies de recherche. Surtout, l'objectivation des projections opérées à son égard peuvent se révéler extrêmement heuristiques. C'est donc autour de ces deux axes - gestion et objectivation des difficultés politiques du terrain - que s'articule notre contribution à 
ce numéro collectif en trois temps, qui furent aussi, schématiquement, les «étapes » du terrain.

Nous montrerons tout d'abord comment la posture de recherche (l'extériorité et la neutralité) définie lors de la construction de l'objet s'est avérée difficile à tenir sur le terrain. Nous verrons ensuite comment l'objectivation des demandes des enquêtés qui rendaient cette posture intenable a révélé le fonctionnement de la ressource "savoir » et finalement la structuration du champ de l'enquête. Enfin, l'étape suivante du terrain - le dépassement des discours identitaires par l'étude de configurations sociales précises - s'est heurtée à de nouvelles projections, cette fois d'ordre sécuritaire. En quoi ces différentes contraintes interfèrent-elles non seulement sur le terrain, mais aussi sur la phase de restitution, une fois la recherche terminée?

Entre négation officielle et surdétermination identitaire : l'objet alévi

Les alévis sont un groupe confessionnel hétérodoxe ${ }^{2}$ que l'on estime constituer entre 15 et $20 \%$ de la population de Turquie. Ils sont donc numériquement d'un ordre de grandeur comparable à celui des Kurdes de Turquie. Toutefois, tandis que ces derniers ont fait l'objet de nombreuses études dans différents champs disciplinaires ${ }^{3}$, les alévis n'ont pas été l'objet d'un tel investissement scientifique. Ce groupe reste d'autant plus difficile à appréhender que l'unitarisme résolu de la Turquie le rend statistiquement invisible 4 . Sa spécificité est niée ou traitée à la marge sur un mode folkloriste par les autorités - pour lesquelles il existe un seul islam en Turquie, l'alévité représentant tout au plus un fait culturel ${ }^{5}$. Cette invisibilité déterminée par l'extérieur se double d'une pratique de dissimulation largement utilisée par les alévis pour échapper à d'éventuelles mesures discriminatoires ${ }^{6}$. Ce mystère a suscité et suscite encore, chez la quasi-totalité des "spécialistes de l'alévité ", une excitation motivée par l'inconnu, le secret, et le mythe de la « découverte » d'un terrain pas, ou si peu, défriché.

Il serait cependant erroné d'affirmer que les alévis ont été peu étudiés. S'ils l'ont été, c'est sur un mode spécifique, et principalement autour de disciplines précises des sciences humaines et sociales. Pour l'essentiel, les études sur les alévis ont été l'apanage d'historiens et surtout d'anthropologues ${ }^{7}$. Cette absence des politologues et sociologues dans les travaux scientifiques sur les alévis est d'autant plus singulière que, sur le terrain des études kurdes, ils dominent. L'absence d'un mouvement politique alévi ayant recours à des répertoires d'action violents et porté sur la scène internationale (par la migration, l'investissement d'arènes internationales ou la médiatisation) est l'une des variables explicatives de cette configuration disciplinaire : à la différence des Kurdes, les alévis sont en général considérés non comme un groupe " politique », mais comme un groupe « religieux » ou « culturel ».

Pourtant, depuis la fin des années 1980, un mouvement identitaire alévi est apparu ${ }^{8}$. En exhibant pour la première fois l'alévité sur un mode souvent spectaculaire, ce dernier a modifié les conditions de recherche sur les alévis et mis fin, au moins en partie, au mystère qui les entourait. L'intérêt croissant pour l'alévité a paradoxalement remis au goût du jour, voire suscité des travaux visant à décrire et à définir un système de croyance ${ }^{9}$. Paradoxalement donc, dans ce contexte nouveau, la curiosité s'est accrue mais les modes d'approche des alévis ont peu changé.

A mon sens, l'insuffisance de ces approches découle d'une occultation des dimensions proprement politiques du phénomène qui s'accompagne d'une double naturalisation: tout d'abord, on prend couramment la " communauté » comme un donné et un tout, ce qui paraissait pourtant loin d'être évident en présence d'une grande diversité et d'une 
sécularisation avancée. En outre, de nombreux travaux considèrent le mouvement aléviste comme allant de soi, exprimant tout haut ce que les alévis pensent tout bas; la tonalité majoritaire reste qu'une "identité » autrefois opprimée parvient enfin à s'exprimer $^{10}$, grâce à la libéralisation politique ainsi qu'à l'essor des médias ${ }^{11}$.

Dès lors, il s'agissait pour nous de renverser la question et d'expliquer pourquoi l'identitaire devient, à un moment donné, un registre pertinent de mobilisation politique. Par rapport à la majorité des études sur les alévis, notre analyse entendait se déplacer de l'architecture de l'histoire des unités sociales et des formes symboliques vers celle de la dynamique historique et politique de leur production et de leur reproduction. Loin de "politiser la question alévie", cette approche de science politique devait dans mon esprit désessentialiser et dévoiler les ressorts politiques des discours et mobilisations identitaires ${ }^{12}$.

De cette problématique découlait aussi la posture de recherche que je choisis d'adopter : la neutralité par rapport au mouvement alévi, l'indépendance critique vis-àvis des positions étatiques certes, mais aussi de ceux qui allaient être mes interlocuteurs. Etant moi-même peu enthousiaste au sujet de ces développements "identitaires ", mais ressentant une certaine sympathie pour l'initiative, je refusai de lui appliquer toute approche normative pour laisser la place à la compréhension.

L'extériorité au terrain et à ses enjeux, atout ou désavantage ?

Mon extériorité objective aux enjeux du mouvement identitaire et ma qualité d'étrangère devaient faciliter l'application de cette indépendance critique à ma relation au terrain. L'extériorité et la dissemblance ont dès lors été constitutives de ma démarche empirique. La différence n'était pas seulement un principe de réalité objectif, mais une stratégie de recherche déclinée en plusieurs points : la non-appartenance à l'alévité, la nationalité française ${ }^{13}$, enfin le fait d'être une femme interrogeant principalement des hommes. Ajoutée à la distance créée par la perception du statut social du chercheur (et des différents capitaux qui lui sont liés), l'accumulation de ces différences, peut générer méfiance, voire hostilité. Mais une telle position présente aussi l'avantage pour son détenteur de ne pas devoir se justifier, se définir ni se situer à l'intérieur du groupe étudié, car il reste indiscutablement autre. Les frontières ne sont dès lors plus un sujet de négociation entre enquêteur et enquêté ${ }^{14}$ : elles sont reconnues et maintenues de part et d'autre.

A cela s'ajoute une particularité de l'alévité qui distingue mon expérience de celle qu'ont pu faire de nombreux chercheurs sur le monde musulman ou d'autres terrains « confessionnels » : je n'ai jamais été confrontée au prosélytisme. Celui-ci n'est pas un mode pertinent d'interaction, dans la mesure où l'appartenance à l'alévité est déterminée par la naissance et ne s'acquiert pas par conversion ${ }^{15}$. Tout au plus, les invitations à devenir alévie par alliance ou par "convergence philosophique» répondaient plutôt, de la part de mes interlocuteurs, à une volonté de créer un lien autour de valeurs considérées comme partagées, et ne remettaient pas en cause mon extériorité. Plus qu'en termes de conviction religieuse ${ }^{16}$, la distinction alévi / chercheur non alévi faisait sens en tant que cadre de présentation de soi $^{17}$. S'il ne s'agissait pas de me faire devenir l'une des leurs, il n'en était pas moins fondamental pour mes interlocuteurs de se présenter à l'enquêteur étranger sous un jour favorable.

Travailler sur un mouvement identitaire : positionnement problématique et neutralité illusoire 
En dépit d'une extériorité revendiquée par moi-même et acceptée par mes interlocuteurs, cette distance critique s'est avérée très difficile à tenir sur le terrain, et source d'inconfort dans l'interaction. Aux yeux de mes premiers interlocuteurs - les cadres associatifs alévis, en Allemagne et en Turquie et, dans une moindre mesure, les membres de ces associations - j'apparaissais certes comme extérieure, mais avant tout comme chercheuse. Mon intérêt scientifique pour le mouvement alévi était compris comme un engagement, au sens large du terme, en sa faveur. Comme beaucoup de mouvements nationalistes ou identitaires ${ }^{18}$, l'alévisme passe par la redécouverte des origines et la réécriture de l'histoire du groupe. Pour ce faire, les entrepreneurs identitaires recherchent la caution d'institutions respectables, en particulier des universités. Ils en attendent non seulement une légitimation de leurs discours, mais aussi qu'elles participent à sa production et à sa transmission, et crédibilisent le mouvement ${ }^{19}$. Le discours scientifique apparaît alors comme une véritable ressource stratégique, à la fois comme miroir symbolique (dans les constructions identitaires) et comme instrument de légitimation (par la reconnaissance "savante »). Le chercheur bénéficie de par son statut d'une position sociale de prestige, convertible en pouvoir. Déstabilisé de sa position d'observateur externe, il est absorbé dans les processus d'auto-objectivation dans lesquels est engagé le mouvement étudiée ${ }^{20}$. Certains interlocuteurs attendaient de ma recherche qu'elle légitime la mythologie identitaire alévie ${ }^{21}$. Il m'a fallu accepter que l'extériorité ne permettait pas ici de prévenir l'engagement, bien au contraire. La reconnaissance et la force du mouvement alévi dépendent en effet pour partie de sa légitimation en tant que sujet collectif dans les arènes politiques et médiatiques internationales, à laquelle il ne peut accéder qu'au prix de l'objectivation de son altérité. C'est donc précisément la proximité entre la posture de recherche et la quête identitaire et politique du mouvement observé qui pose problème et fausse l'idéal d'objectivité.

Si cette demande de légitimation adressée au chercheur peut paraître sympathique, elle n'en reste pas moins susceptible d'analyse et pose un véritable problème déontologique. Confronté à une telle situation, le chercheur a-t-il une obligation d'engagement ou une obligation de réserve? L'engagement du chercheur est-il envisageable comme un choix éthique ou politique individuel, qui serait extérieur au projet scientifique? Est-il au contraire une dimension constitutive de la construction de l'objet et de la relation au terrain? Dans quelle mesure l'engagement du chercheur devient-il à la fois cadre et condition de sa recherche ? L'idée de neutralité scientifique est-elle un escamotage des rapports de domination qui rendent possible l'intrusion du chercheur? Existe-t-il une "responsabilité professionnelle» de l'engagement sous peine de s'associer à la domination ?22

Certains chercheurs sur les alévis ont cautionné ou reproduit des discours identitaires essentialisants sans les questionner. La relative bienveillance du monde académique occidental envers les alévis s'explique en partie par la sympathie que ces derniers inspirent: ils sont globalement pauvres, opprimés et souvent syndiqués. Cette sympathie s'étend souvent à l'alévisme, notamment en raison de son caractère " politiquement correct ». A la différence de certains pans du mouvement nationaliste kurde, le mouvement aléviste n'est pas armé, n'utilise pas la violence et ne remet pas en cause les frontières. Ses revendications - liberté de religion, protection de la laïcité en Turquie, protection des minorités - sont largement considérées comme légitimes. Les alévis ont surtout bien su répondre aux attentes de certaines sphères académiques et politiques, notamment européennes ${ }^{23}$. Ils s'affichent comme défenseurs du 
kémalisme et des droits de l'homme, toujours «victimes » et opprimés par le régime comme par les islamistes. S'énonçant dans la majorité des situations comme démocrates et modernes "par essence ", ils sont devenus, pour beaucoup, le meilleur rempart contre ce que l'Occident comme le régime turc diabolisent souvent, l'islamisme - voire l'islam.

L'exemple des alévis nous semble illustrer pertinemment la rencontre entre des projections occidentales - et notamment des sphères académiques - et les intérêts politiques d'un mouvement. Le monde académique occidental a parfois reproduit la vulgate historique alévie ${ }^{24}$, faisant de ses protagonistes les «alliés de la laïcité » ou les " fers de lance de la démocratie $»^{25}$, peut-être pour des raisons tout aussi idéologiques ou politiques que celles de leurs confrères alévistes. Cette logique est particulièrement visible au niveau de ce que je qualifie de "biais féminin ». Se définissant en miroir de l'islam sunnite qu'ils contribuent à diaboliser, de nombreux alévis proclament pratiquer l'égalité des sexes et bien traiter les femmes ${ }^{26}$. Cet argument est ensuite mobilisé aussi bien vis-à-vis de la Turquie kémaliste que des partenaires occidentaux. Mes interlocuteurs ont ainsi souvent mis en avant ma qualité de femme pour me prendre à parti, pensant alors me dire ce que je souhaitais entendre. Cette « ressource féminine " qui me permettait de créer un lien avec certaines de mes interlocutrices était en partie neutralisée par son absorption dans le discours identitaire. Malgré moi, je me trouvais entraînée précisément dans les dynamiques que je voulais dénoncer, et dont je commençais à saisir les logiques.

Accepter qu'une réflexion scientifique soit implicitement inféodée à la reproduction d'un discours identitaire engage une compromission contraire à tout projet de connaissance ${ }^{27}$. D'un point de vue déontologique, la distance indispensable à l'analyse n'existe plus quand le discours scientifique et l'idéologie des enquêtés se confondent. La distinction classique entre le savant et le politique prend ici tout son $\operatorname{sens}^{28}$ : les logiques professionnelles du militant et du chercheur, et les éthiques - donc les positionnements axiologiques - qui les accompagnent, doivent rester distinctes. En ce sens, je refusais toute homologie - que mes interlocuteurs au contraire encourageaient - entre ma position et celle des intellectuels engagés ou exégètes identitaires. Cette posture est évidemment aussi le fruit d'une socialisation précise. Plus que le mouvement aléviste, le milieu universitaire européen en sciences sociales constituait mon ancrage - ce qui correspondait aussi à un projet professionnel dont les alévis ne constituaient pas le pivot. Pour éviter d'y être discréditée, les procédures de contrôle de ce milieu, implicites il est vrai, étaient pour moi plus contraignantes que celles du mouvement.

Nonobstant une certaine empathie pour des interlocuteurs ouverts et accueillants, j'ai donc choisi de garder une distance face à leur engagement. Dès lors, j'ai dû adapter mes méthodes d'enquête et ma relation au terrain dans son ensemble aux difficultés à maintenir mon extériorité. Pour construire une analyse relativement autonome des discours des enquêtés, il me fallait étudier le milieu produisant des discours et pratiques ritualisés, faire des interprétations des acteurs des données à interpréter, et incorporer dans la construction même de l'objet cette "demande sociale». Cette démarche, qui n'avait pas été planifiée, découlait des dynamiques déclenchées par l'enquête elle-même.

Cette tentative d'objectivation n'a pas tardé à me placer en porte-à-faux par rapport à mes interlocuteurs, pour qui mon travail était compréhensible et légitime tant qu'il 
reproduisait un discours apologétique ou qu'il aspirait à dévoiler l'essence de l'alévité. Mon approche divergeait profondément de leurs attentes: je n'ambitionnais pas de chercher la vérité sur les alévis; je ne m'intéressais pas aux questions qui les préoccupaient, et je voulais précisément sortir de cette vision par l'origine et par l'essence dont ils étaient si férus. Ce qu'ils tenaient pour leur identité, leur histoire, je le traitais comme discours, comme construction d'un récit, comme tentative d'organiser une mémoire ${ }^{29}$. La difficulté tenait à ce que la demande sociale qui justifiait ma présence à leurs yeux conditionnait le déroulement même de l'enquête.

Ce décalage a entraîné l'impossibilité de jouer cartes sur table. Dans nombre d'interactions, il m'a fallu feindre l'ignorance ou l'accord, mentir par omission ou passer sous silence certains registres d'informations, pour des raisons de confidentialité, mais aussi par stratégie de savoir. Tout en écoutant - en simulant l'étonnement, voire en acquiesçant - des discours déjà entendus à maintes reprises, et auxquels j'étais loin de croire, j'essayais d'en dégager l'éventuelle spécificité, la pertinence situationnelle ou les déterminants sociologiques. Certains informateurs avaient besoin de sentir une approbation de ma part pour se livrer. Comme d'autres chercheurs avant moi, j'ai dû m'efforcer de me comporter conformément à ce (que je pensais) que les enquêtés attendaient de moi, user d'un double langage pour leur inspirer confiance et essayer de «les faire parler » un maximum. Dans les situations d'enquête, je ressentais constamment une tension entre la volonté d'expliquer mon projet aux enquêtés et la conscience d'une compréhension impossible ou la peur de me fermer des portes, entre une honnêteté intellectuelle et morale d'une part et la tentation du mensonge pour répondre à leurs attentes de l'autre. Refusant les rôles d'avocate et de relais, j'ai le sentiment d'avoir trahi la confiance que m'ont accordée mes interlocuteurs, souvent gagnée à coups de dissimulation. Ce décalage devenait particulièrement douloureux à mesure que mon implication personnelle s'intensifiait, que se créaient des relations de proximité avec certains de mes interlocuteurs, jusqu'à,parfois, «faire tomber le masque » quand la confiance était assez solidement installée.

Ce positionnement problématique s'est également révélé particulièrement difficile à tenir quand certains de mes interlocuteurs m'assignaient des rôles qui confinaient aux limites de ma posture. Je pense à des demandes de soutien et d'aide en tout genre (signature de pétitions, aide financière) auxquelles j'ai toujours opposé une fin de nonrecevoir. Certains m'ont adressé des demandes d'expertise ou de conseils plus «politiques». De manière informelle ou dans des contextes plus officiels et contraignants ${ }^{30}$, on $\mathrm{m}^{\prime} \mathrm{a}$ demandé mon analyse du mouvement ou les options stratégiques que je privilégierais. D'autres encore m'ont conféré le rôle de conseiller dans la production d'un argumentaire politique destiné à faciliter l'accès aux processus de décision politique et aux ressources publiques, financières ou symboliques ${ }^{31}$. Ce rôle d'expert apparaissait d'autant plus séduisant qu'il pouvait me permettre d'observer de l'intérieur, d'avoir accès à des informations de bonne qualité, car l'intérêt des informateurs était directement en jeu ${ }^{32}$. Mais je pouvais alors être prise au piège et voir mon travail servir à transformer la situation dont il se voulait le témoin. Cette situation particulière rejoint des enjeux déontologiques plus généraux. Dans quelle mesure le chercheur contribue-t-il à construire un objet, susceptible d'être ensuite repris non seulement par les médias, mais par les acteurs politiques eux-mêmes? Même si mon intervention n'aurait probablement pas produit d'effets politiques directs et sensibles, j'ai refusé d'endosser ces rôles. 
Ces refus entrainaient cependant une perte de visibilité de mon projet pour les enquêtés, qui risquait de m'aliéner leur confiance. Eviter de répondre à ce genre de sollicitations nécessitait donc une justification de ma part. J'ai dû employer plusieurs tactiques pour expliciter mes choix, pas toujours couronnées de succès. Je me présentais en général comme une novice, pas encore assez savante pour endosser ces rôles. Au fur et à mesure des progrès de mon travail, cet argument perdait naturellement en crédibilité. Un autre motif que j'ai employé pour échapper aux contributions financières fut la légendaire pauvreté des étudiants. Un dernier argument que j'ai mobilisé concernait mon extériorité au mouvement : «En quoi ai-je le droit de me prononcer sur l'alévisme, moi qui ne suis pas alévie? C'est à vous de savoir. Je ne peux pas vous dicter de l'extérieur ce que vous devez faire... ».

Cette extériorité - que j'estimais être un gage d'impunité et de non-engagement - s'est vite révélée un élément dont les usages sociaux étaient plus complexes que je ne l'imaginais au début de la recherche. Mon positionnement était en effet problématique non seulement au regard de mon refus de l'engagement ou de faire l'apologie du mouvement dans les sphères occidentales, mais aussi sur le plan de la connaissance, champ dans lequel je me déplaçais principalement. Même imprévue et difficile à gérer, la confrontation répétée avec les acteurs identitaires m'a fait percevoir puis comprendre la structuration du champ du savoir alévi, ses enjeux et mécanismes, et la place particulière qu'y occupe le chercheur étranger. Outre la "substance" des entretiens, côtoyer ce milieu, objectiver les projections auxquelles les enquêtés se livraient sur moi-même m'a permis d'en repérer les configurations d'acteurs, d'en déceler les logiques et les régimes de justification. En ce sens, l'inconfort méthodologique s'est in fine révélé heuristique.

Le dévoilement de la structure du champ aléviste

Le champ alévi présente une structure particulière que je saisissais au fur et à mesure que j'objectivais la manière dont j'y étais absorbée malgré moi à travers l'un de ses enjeux centraux, le savoir. Dans le courant du mouvement, aucun consensus n'a émergé sur l'origine ni sur la nature même de l'alévité. Certains acteurs interprètent cette dernière comme un fait religieux, par exemple comme la forme spécifiquement turque de l'islam, comme « le véritable islam » débarrassé de ses influences arabes, ou encore comme religion sans rapport avec l'islam, donc véritablement nationale. D'autres conçoivent au contraire l'alévité comme un fait politique. Ils la définissent comme inscrite dans « la tradition de la démocratie » ou comme la traduction d'une «culture de la rébellion $\aleph^{33}$. D'autres encore soulignent sa dimension ethnique, faisant de l'alévité un support de la turcité ou l'assimilant à un phénomène authentiquement kurde... Les derniers se contentent d'en faire une «culture » incluant - suivant des dosages variables - ces différents aspects. Inutile de préciser qu'entre ces différents exégètes, les débats sont très virulents. C'est en effet, à travers le sens du phénomène, l'inscription de l'alévité dans la nation, et les catégories de négociation et de revendication de l'altérité, qui sont in fine en discussion.

La persistance de ces débats et la non-attribution d'un sens fixe à l'alévité ne s'expliquent qu'à la marge par le caractère syncrétique du phénomène. Elles sont avant tout dues au fait qu'au cours du mouvement aucun acteur de référence n'a émergé, susceptible d'imposer une interprétation unique de l'alévité dans des catégories politiquement pertinentes. Cela tient à la fois à la configuration d'acteurs (aucun n'étant assez puissant par rapport aux autres pour imposer sa manière de voir) et à 
l'absence d'institutions permettant d'objectiver le savoir, ou même d'une source de savoir acceptable et acceptée par tous.

L'enjeu du savoir et la structuration du champ ${ }^{34}$

Dans ces conditions, le savoir n'est pas seulement un capital symbolique. Il constitue une ressource identitaire de premier plan, pour le mouvement en général, mais aussi pour chaque acteur pris en particulier ${ }^{35}$. Dans ce contexte, l'histoire est considérée comme une «technique " permettant de dévoiler l'origine, et donc le sens de l'alévitée ${ }^{36}$. Le savoir « vrai » serait susceptible de dévoiler la définition réelle de l'alévité, de percer sa véritable nature ${ }^{37}$. C'est la raison pour laquelle toutes les organisations alévies accordent une importance centrale à la connaissance et à la recherche ${ }^{38}$. En 1997, une "Académie européenne alévie » est créée, avec pour ambition de "refonder le savoir alévi ». Un maître de conférences militant projettera même de fonder une "sociologie alévie » comme discipline séparée ${ }^{39}$. Les différentes organisations rivalisent pour former la meilleure bibliothèque et pour s'attacher les bonnes grâces des scientifiques... La constitution d'un savoir « sérieux » sur l'alévité est l'une des priorités du mouvement. Quelle que soit la conception de l'alévité défendue, la conviction que le savoir historique permettra de régler les divergences est unanimement partagée.

Acteurs, producteurs et consommateurs du mouvement alévi ont par ailleurs conscience de leur désaccord sur la nature même de l'alévité. Si tous sont à la recherche d'un savoir pertinent, les récits entendus sont si contradictoires qu'ils ne savent souvent pas qui croire. Lors des panels organisés par les associations alévies, au cours desquels des exégètes défendent des conceptions différentes de l'alévité, les questions posées par l'assistance reviennent avec une régularité déconcertante: «ce que vous avez dit est très intéressant. Mais il y a une chose que j'aimerais savoir: faisons nous partie de l'islam, oui ou non?». Et le débat reprend de plus belle... Rares sont ceux qui rentrent chez eux avec une ready-made answer. Cette situation génère une insatisfaction par rapport aux associations et aux exégètes jugés «incompétents ». Ces divergences sont généralement considérées comme résultant de deux facteurs principaux. Le premier est une lacune de savoir : si les spécialistes ne sont pas d'accord, c'est qu'ils ont mal étudié. On les excuse en partie en admettant que les sources alévies ont été détruites par les Ottomans ou que l'entreprise de construction d'un savoir alévi est nouvelle.

Mais qui détient ce précieux savoir? Traditionnellement, le savoir alévi, oral et principalement religieux, était incorporé et monopolisé par les lignages sacrés, les dede. Cette chaîne de transmission ayant été largement interrompue depuis l'exode rural massif, la descendance sainte, même si elle est toujours respectée, n'est plus une garantie de savoir. Avec l'alphabétisation massive, le mouvement aléviste - qui s'est accompagné d'une importante scripturalisation du savoir - a permis une redistribution du capital-savoir. "Dans les sociétés sans écriture, le savoir hérité ne peut survivre qu'à l'état incorporé. En détachant les ressources culturelles de la personne, l'écriture(...) permet l'accumulation de la culture jusque-là conservée à l'état incorporé et, corrélativement, l'accumulation primitive du capital culturel(...)à travers(...)les instruments d'appropriation de ces ressources(écriture, lecture) $»^{40}$. Avec l'éducation de masse, on assiste ainsi à une démocratisation de l'accès, mais aussi de la production du savoir sur l'alévitée ${ }^{41}$.

Assiste-t-on pour autant à l'apparition d'un nouveau corps d'exégètes, comparable au clergé, c'est-à-dire un groupe détenteur du monopole de la manipulation des visions du monde $^{42}$ ? Une figure nouvelle du savoir scriptural, le araștırmact-yazar ${ }^{43}$, érudit 
local,est apparue. Il ne s'agit ni de membres de lignages sacrés, ni de scientifiques au sens propre, mais d'enseignants ou de journalistes qui se sont improvisés exégètes de l'alévité. Ce label désigne autant le «savant du quartier » prompt à sortir sa plume pour remplir la feuille de chou locale de ses idées sur la question et à exhiber avec fierté les quelques livres qu'il prétend avoir lus, que des personnalités considérées comme des références, et qui publient des ouvrages ou des articles journalistiques ${ }^{44}$. Leurs écrits reposent souvent sur des connaissances scientifiques, historiques ou théologiques faibles et rarement sur des recherches de terrain ${ }^{45}$. Ils s'apparentent souvent à un travail de "compilation » d'ouvrages similaires déjà parus sur la question, qui peut aller jusqu'au recopiage de pages entières, sans pour autant citer de références ${ }^{46}$.

Les araşttrmact-yazar sont pourtant loin d'avoir monopolisé ou même hiérarchisé le champ du savoir alévi. Cette ressource n'est pas institutionnalisée: elle ne dispose d'aucun "diplôme", d'aucune garantie de sérieux. A l'augmentation des enjeux (identitaires et politiques) liés au savoir alévi s'ajoute la multiplication et la nonhiérarchisation de ses sources et de ses porteurs. Les querelles font évidemment rage entre araştırmact-yazar et dede. Les premiers accusent les derniers de ne connaître que quelques vieilles légendes, transmises on ne sait comment, et rationnellement intenables; les seconds rétorquent que l'on n'apprend pas l'alévité dans les livres... Mais les querelles éclatent tout autant entre dede. Ces derniers se contestent mutuellement la qualité de dignitaires religieux, en dénonçant par exemple les références « insuffisantes » au Coran des autres, leur « méconnaissance » de telle prière ou leur conduite « erronée » des cérémonies. Pas plus que les dede, les araștırmact-yazar ne constituent un groupe hiérarchisé : parce qu'ils défendent des positions différentes sur la nature de l'alévité, ces derniers se soupçonnent mutuellement de «ne rien y connaître ». On assiste donc à une véritable dérégulation, au sens de "l'évidement des dispositifs institutionnels de la régulation du croire $»^{47}$. Cette dérégulation s'accompagne d'un processus de subjectivisation, lorsque l'acteur se constitue en «sujet » d'action et maitre de catégories réifiées. Il utilise alors ces dernières pour construire une voie vers la vérité, et transcende ainsi l'identification statique avec des catégories objectivées de l'identité collective ${ }^{48}$.

Mais le fait que chacun s'autoproclame savant sur la question brouille les limites même du savoir. De fait, «la place faite aux stratégies symboliques ne peut qu'aller croissant, comme les chances d'efficacité qui leur sont objectivement imparties, lorsqu'on va des sociétés où(...)les manifestations de la différence sont régies par de véritables lois somptuaires, à des univers sociaux où l'indétermination de la valeur objective de la valeur fiduciaire autorise et favorise la prétention(...)et les stratégies de bluff par lesquelles elle vise à se réaliser $»^{49}$. Le désaccord patent entre savants autoproclamés renforce tendanciellement cette situation d'incertitude et l'appel à un savoir «vrai » consensuel, fondé, mais malheureusement occulté... Le savoir sur l'alévité n'est pas naturalisé ni objectivé. Alors qu'il représente une ressource essentielle pour tous les acteurs identitaires et est sacralisé dans l'absolu, son bien-fondé peut toujours être remis en question. Au lieu d'aboutir à l'unification du mouvement, le savoir contribue donc à la dispersion des interprétations et à la multiplication des conflits.

La seconde explication couramment apportée au désaccord entre spécialistes sur la nature de l'alévité invoque leur supposée subjectivité. Il leur est reproché de ne pas écrire de manière objective, mais d'orienter leurs recherches en fonction de convictions politiques ou d'intérêts bassement carriéristes. Entre tenants de versions 
rivales de l'alévité, on s'accuse à la fois de "ne pas savoir de quoi on parle» et "d'interpréter les données de manière idéologique et orientée ».

\section{NOTES}

1. . Cette recherche menée en Turquie et en Allemagne entre 1999 et 2002 s'inscrit dans le cadre d'une thèse de doctorat intitulée Construction identitaire, mobilisation et territorialité politique : le mouvement aléviste en Turquie et en Allemagne depuis la fin des années 1980 et soutenue à l'Institut d'études politiques de Paris en octobre 2002 (direction de thèse : Gilles Kepel).

2. . En raison des éléments d'origine chiite de leur culte (adoration d'Ali et des douze imams, jeûne de Muharrem...), les alévis sont parfois considérés comme des « chiites extrêmes ». Cependant, en raison de leur négligence des piliers de l'islam et de pratiques probablement antérieures (culte des arbres, des pierres, etc.) qu'il reste difficile d'attribuer à une origine précise, les alévis ne sont pas considérés par les chiites orthodoxes comme chiites. Quant aux sunnites, ils oscillent entre leur dénier la qualité même de musulmans et les assimiler à l'islam en niant toute spécificité ou en rapportant ces dernières à des déviances désormais caduques. On trouve des groupes qui présentent certains traits similaires dans les Balkans (Kızılbaş en Bulgarie et bektaşi en Albanie notamment) et jusqu'en Iran (les groupes dits Ahl-e Hakk) et en Syrie (proximité avec les alaouites).

3. . Voir notamment les travaux de Martin van Bruinessen et Hamit Bozarslan.

4. . La « particularité » alévie est tue plus encore que d'autres. Jusqu'en 1965, les recensements officiels mentionnaient les langues parlées, reconnaissant implicitement la réalité, au moins linguistique, du fait minoritaire. L'annuaire de statistiques de 1965 consacre également un paragraphe à la répartition confessionnelle des citoyens. Il prend soin de catégoriser les chrétiens en catholiques, orthodoxes, protestants, grégoriens ; mais il ne distingue jamais, pour les musulmans, entre sunnites et alévis. La carte d'identité est théoriquement plus détaillée, prévoyant le double aveu de la religion et de la confession. Mais nous n'avons jamais rencontré d'alévi dont les papiers d'identité portent une autre indication confessionnelle que « sunnite ». Il reste cependant difficile d'attribuer la responsabilité de cette « profession de foi » mensongère au titulaire de la carte ou au secrétaire municipal. Voir Roux J. P., Les traditions des nomades de la Turquie méridionale, Paris, Maisonneuve, 1970, pp. 83-84. 5. . La Direction des Affaires Religieuses, institution vouée à la gestion étatique de l'islam, fait construire des mosquées dans les villages alévis, alors que ces derniers ne les fréquentent pas. De même, les cours d'instruction religieuse obligatoires à l'école ne font aucun cas de la spécificité alévie. D'une manière générale, la Direction considère les alévis comme des sunnites ayant dévié de la juste voie.

6. . Cette dissimulation (takiyye), issue du répertoire chiite, permet de passer sous silence cette appartenance dans les situations où elle peut être préjudiciable.

7. . Voir Gokalp A., Têtes Rouges et Bouches Noires, une confrérie tribale de l'ouest anatolien, Paris, Société d'ethnographie, 1980 ; Kehl-Bodrogi, K., Die Kızılbaş / Aleviten. 
Untersuchungen über eine esoterische Glaubensgemeinschaft in Anatolien, Berlin, Klaus Schwarz, 1988. Pour les historiens, voir notamment les travaux de Jean-Louis Bacqué-Grammont pour les XVème et XVIème siècles. Très peu d'études en revanche sont consacrées à la période postérieure au XVIIème siècle. Voir également les nombreux travaux d'Irène Mélikoff.

8. . Celui-ci reste loin d'épuiser la problématique des mobilisations des alévis, beaucoup plus diversifiées. Durant les années 1970, ces derniers étaient largement engagés à gauche, parfois radicale. L'une des seules contributions sur la dimension politique de la question alévie est Bayart J.-F., "La question Alevî dans la Turquie moderne », in : Carré O. (dir.), L'islam et l'Etat dans le monde aujourd'hui, Paris, PUF, 1982, pp. 109-120.

9. Quelques travaux concernant ce mouvement ont vu le jour, surtout en Allemagne. Ils restent cependant en partie l'apanage des mêmes auteurs spécialistes des alévis, et largement d'anthropologues. Voir Vorhoff K., Zwischen Glaube, Nation und neuer Gemeinschaft : alevitische Identität in der Türkei der Gegenwart, Berlin, Klaus Schwarz, 1995.

10. . «On peut espérer [pour les alévis] et leur communauté que la voie de l'émancipation dans laquelle ils se sont engagés soit une voie de la paix, du respect et de la tolérance », Vorhoff K., Zwischen Glaube..., op cit., p. 5.

11. . Yavuz M. H., « Media Identities for Alevis and Kurds in Turkey », in Eickelman, D., Anderson J. (dir.), New Media in the Muslim World : The Emerging Public Sphere, Bloomington, Indiana University Press, 1999, pp. 180-199.

12. . Dès lors, nous devions distinguer entre " alévité » (le fait sociologique) et " alévisme " (le mouvement politique).

13. . Celle-ci était importante non seulement en Turquie, mais aussi en Allemagne, dans la mesure où elle nous y distinguait de la société majoritaire et créait une sorte de solidarité entre étrangers.

14. . Voir Tietze N., L'islam : un mode de construction subjective dans la modernité. Des formes de religiosité musulmane chez les jeunes hommes dans les quartiers défavorisés en France et en Allemagne, Thèse de doctorat, Paris, EHESS, 1999, p. 51.

15. . Même si ce principe tend à être remis en cause par le courant « moderniste » du mouvement alévi.

16. . D'une manière générale, l'alévité ne relève pas uniquement du religieux, mais d'une appartenance initialement déterminée par la confession, mais qui ne se réduit plus à la dimension religieuse proprement dite.

17. La faible pertinence - pour ne pas dire l'absence - de la question de la croyance au long de l'enquête est également due au fait que j'interrogeais peu cette dimension ; en outre, mes interlocuteurs ont rarement jugé utile de mentionner ce sujet. Dans une certaine mesure, la dimension croyante devient marginale à mesure que tout discours sur l'alévisme est absorbé par la logique identitaire ; même si un discours n'est pas conçu comme tel, il est souvent interprété dans ce cadre.

18. . Voir par exemple Jaffrelot $C$., « Réinterprétation du mythe de Ram et mobilisation nationaliste hindoue », in D.-C. Martin (dir.), Cartes d'identité. Comment dit-on « nous » en politique ?, Paris, FNSP, 1994, pp. 101-121.

19. . Voir par exemple la brochure publiée par la fédération des alévis d'Allemagne, dans laquelle sont mis à contribution divers chercheurs allemands : Vorhoff K. et al., Wie der Phönix aus der Asche, Renaissance des Alevismus, Glaubenslehre, Moderne, Musik, Organisationsformen, Cologne, AABF, 1998. Pour un processus similaire, voir Picard E., « De la domination du groupe à l'invention de son identité. Les milices 
libanaises et les paramilitaires nord-irlandais », pp. 147-162, in D.-C. Martin (dir.), Cartes d'identité..., op. cit., p. 156.

20. . Albert B., « Situation ethnographique et mouvements ethniques. Réflexions sur le terrain post-malinowskien », in Agier M. (dir.), Anthropologues en dangers.

L'engagement sur le terrain, Paris, Jean-Michel Place, 1997, p. 85.

21. . En me proposant pas exemple de publier dans leurs revues.

22. . Moizo B., «L'anthropologie aboriginaliste : de l'application à la fiction », in Agier M. (dir.), op. cit., p. 71.

23. . Mais aussi turques : plusieurs universitaires turcs, notamment kémalistes, présentent un biais pro-alévi évident.

24. . Ce travers est dénoncé avec justesse par Bozarslan H., « L'alévisme, la métahistoire et les mythes fondateurs de la recherche ", in : Rigoni, I. (coordination), Turquie : les mille visages. Politique, religion, femmes, immigration, Paris, Syllepse, 2000, pp. 77-88.

25. . Voir notamment Gökalp A., « Les alevî », in Les Turcs : Orient et occident, islam et laïcité, Paris, Autrement, série monde, hors-série n76, septembre 1994, pp. 112-125 ; Bilici, F., « Les alévis, alliés naturels du kémalisme en Turquie ? » in Bozdemir, M. (dir.), Islam et laïcité : approches globales et régionales, Paris, L'Harmattan, 1996, pp. 281-298. 26. . Ce discours s'appuie sur certains éléments tangibles comme la participation des femmes aux cérémonies religieuses sur un pied d'égalité avec les hommes, l'absence d'obligation de se voiler pour des raisons religieuses (en tout cas de manière moins systématique), l'interdiction de la polygamie et du divorce. Cependant, les alévis relèvent de la même culture patriarcale que la population de Turquie dans son ensemble, et les pratiques diffèrent peu, comme le montre la rareté des femmes parmi les cadres du mouvement aléviste. Que la majorité des chercheurs occidentaux sur les alévis soient des femmes - et que ces dernières soient clairement surreprésentées dans les « études alévies » par rapport à leur nombre parmi les spécialistes de la Turquie n'est certainement pas une pure coïncidence.

27. . Albert B., op. cit., pp. 85-86.

28. . Weber M., Le savant et le politique, Paris, UGE, 1963.

29. . Čapo wegnač J., « Faire de l'ethnologie en Croatie dans les années quatre-vingtdix », Ethnologie Française n³1, janvier 2001, «Terrains minés », p. 44.

30. . Réunion de « brainstorming » d'experts sur les développements du mouvement aléviste organisée par l'Académie alévie européenne à Munich en 2001.

31. . Albert B., op. cit., p. 85.

32. . Moizo B., op. cit., p. 73.

33. . En s'appuyant par exemple sur les révoltes attribuées aux alévis sous l'Empire ottoman ou sur l'engagement massif des alévis dans la gauche dans les années 1970.

34. . En nous inspirant des sociologies de Norbert Elias et de Pierre Bourdieu, nous concevons un « champ » comme un contexte social spécifique, espace structuré et conflictuel de positions autour d'un enjeu commun, ici la détention du savoir sur l'alévité. Ce champ du savoir est lui-même partie du champ identitaire alévi.

35. . On pourra se reporter à notre « Local Productions of Alevi Knowledge ", Istanbuler Almanach, 5/2001, pp. 70-74.

36. . Bozarslan H., op. cit.

37. . Cette tendance s'inscrit dans un imaginaire plus vaste de la société turque, qui considère le sociologue comme quelqu'un devant trouver des réponses adéquates aux problèmes sociaux, conformément à une représentation rationaliste de l'intellectuel 
comme leader apte à guider le peuple. La sociologie doit produire des recettes « scientifiques », donc miracles, aux problèmes sociaux. Özdalga E., « Türkiye'de Bir Sosyolog Olmak Üzerine », Toplum ve Bilim n 48-49, 1990, pp. 27-33.

38. . Dès 1993, une revue alévie appelle à la fondation d'une archive de textes religieux et de documents alévis. Kervan $n^{\circ} 25,1993$, p. 19. Dès 1993 également, la fédération alévie d'Europe projette de fonder un institut alévi. La fondation Ehl-i Beyt appelle à créer des « universités Ehl-i Beyt ».

39. . Naturalisé et déterminé par une essence, le groupe devient alors à la fois source et objet d'une sociologie à part (et non pas d'une sociologie des alévis ou de l'alévisme). Voir Bal H., Alevi-Bektaşi sosyolojisi, Istanbul, Ant, 1997. Cet ouvrage définit les rapports qu'une telle science entretient avec les autres sciences, envisage la manière dont elle pourrait s'intégrer dans le cadre universitaire, et propose des domaines de recherche précis.

40. . Bourdieu P., Le sens pratique, Paris, Minuit, 1980, p. 123, 214-215.

41. . Laïdi Z., « Introduction : La lente émergence d'espaces de sens dans le monde ». In : Laïdi Z. (dir.), Géopolitique du sens, op. cit., p. 21. Cette évolution se rapproche de celle constatée dans l'islam orthodoxe parmi les mouvements islamistes, l'accès aux textes sacrés pour toute une génération permettant la remise en cause du monopole de l'interprétation des textes. Kepel G., Richard Y. (dir.), Intellectuels et militants de l'Islam contemporain, Paris, Seuil, 1990.

42. . Pour reprendre des termes wébériens.

43. . Mot à mot, chercheur-écrivain. Ce phénomène n'est pas exclusif aux alévis, mais il acquiert au sein du mouvement alévi des dimensions particulièrement importantes.

44. . Pour un aperçu assez complet et sans complaisance de nombreux ouvrages de cette vague, voir Vorhoff K., " Academic and Journalistic Publications on the Alevi and Bektashi of Turkey », in Olsson T., Özdalga E., Raudvere C., Alevi Identity, Istanbul, Swedish Research Institute, 1998, pp. 23-50.

45. . Si ce n'est quelques anecdotes rapportées du village...

46. . Cela explique la forte intertextualité qui caractérise les productions identitaires alévistes.

47. . Hervieu-Léger D., « Renouveaux religieux et nationalistes : la double dérégulation », in Birnbaum P. (dir.), Sociologie des nationalismes, Paris, PUF, 1997, pp. 163-185.

48. . Salvatore A., Islam and the Political Discourse of Modernity, Reading Ithaca Press, 1997, p. 22.

49. . Bourdieu P., Le sens pratique, op. cit., p. 240. La vision interactionniste de l'auteur se réfère ici à un univers social à très faible degré d'institutionnalisation du capital symbolique, aux hiérarchies multiples, brouillées et changeantes, et donc porteur d'une incertitude objective, vision applicable au champ du savoir alévi. 
INDEX

Index géographique: Turquie

Mots-clés : sociologie 